\title{
Sukzession in der früh-modernen Literatur der Sant-Bewegung am Beispiel von Kabir (gest. 1518) und Sundardas (1596-1689)
}

\begin{abstract}
This article explains the transmission of religious authority in the context of the early-modern Indian movement known as the Sant tradition. This study uses emic categories from two contrasting examples to highlight the limits and possibilities of transcultural comparative approaches. In the first case of Kabir, a weaver-poet, authority is based on the realization of the divine revelation within himself and the capacity to render this experience orally. Succession to leadership is built by his followers, who against Kabir's own perspective, organize institutionalized affiliations that take the shape of schools, often with initiation. In the second case of Sundardas, a second generation Sant in the line of Dadu (the recognized guru and founder of the group), authority is presented through his erudition and capacity to consolidate the group with a religious identity inside Hinduism. Succession is assured by affiliation through initiation and highlights the importance of writing to foster a group identity. As a heuristic tool, comparison proofs fruitful when applied not to generalizing concepts but to specific cases and themes.
\end{abstract}

Wie überlebt eine mündliche Lehre eines Meisters, der keiner sein will? Wie kann Schriftlichkeit Autorität verleihen, wenn diese sich nur aus direkter Erfahrung des Göttlichen ableitet? Kabir und Sundardas, ${ }^{1}$ zwei vormoderne Dichter, werden hier als Beispiele religiöser Tradierung angeführt. In den verschiedenen religiösen Strömungen Indiens, die man unter der Bezeichnung Hinduismus zusammengefasst hat, kann die Einrichtung des Guru als einer heiligmäßigen Person eine entscheidende Rolle in der Weitergabe religiöser Inhalte spielen. ${ }^{2}$ Ein wichtiger Aspekt der Sukzession dreht sich um diese zentrale Figur, welche mündlicher Exponent ihrer Ideen und oft Ausgangspunkt schriftlicher Überlieferungen ist. Diese Gestalt ist jedoch Teil eines breiteren Bereichs von Personen, die an

1 Die Eigennamen werden ohne Diakritika geschrieben.

2 Zur Figur des Guru in der indischen Geschichte: Steinmann, Guru Śișiya Sambandha; in der Sant-Tradition: Gold, Lord as Guru; in der Moderne: Suthren Hirst und Zavos, Religious Traditions. 
der Weitergabe religiöser Inhalte mitwirken, ${ }^{3}$ die allerdings bezüglich Zeit und Lokalisierung auf dem indischen Subkontinent so unterschiedlich sind, dass es keine einfache und verallgemeinerbare Antwort auf die Frage nach der Rolle des Guru und die Art, wie Sukzession funktioniert, gibt. ${ }^{4}$ Ich werde mich auf zwei Gestalten aus der Sant-Bewegung konzentrieren, die Verfahren und Möglichkeiten aufzeigen, wie Bezug auf eine Tradition verwirklicht bzw. wie sie mit einer neuen Botschaft verändert wird.

Der Ausdruck »Sant« bezieht sich erst im Nachhinein auf Kabir und die ihm Gleichgesinnten und wird dadurch zur Bezeichnung der Gruppe oder Bewegung jener, die die Vorstellung von einem Absoluten, einem höchsten Wesen ohne Eigenschaften (nirguna), gegenüber einer personifizierten Gottheit bevorzugen. Ihre Spiritualität sollte ausschließlich nach innen gewandt sein, wo sie die Antworten auf ihre Suche erleben. Nach der wörtlichen Bedeutung ist ein Sant eine Person, welche das »Seiende, Wirkliche« (sat) verkörpert und wahr(haftig) ist. Obwohl schwer auszumachen ist, wann in der Epoche Kabirs das Wort gebräuchlich wurde: Heutzutage ist es die übliche Bezeichnung für die Anhänger einer Bewegung, welche diese charakteristische Botschaft verbreitet. Viele Elemente, die heutzutage auf Sants zutreffen, sind in den Liedgedichten Kabirs enthalten: Ein Sant ist jemand, der spirituelle Erleuchtung oder Selbstverwirklichung, also Befreiung in diesem Leben erreicht und erfahren hat. Sant kann folglich den Schüler oder Jünger eines Sant-Meisters bezeichnen, der auf dem Weg zur Verwirklichung von dessen Botschaft ist. Sants gibt es unter Hindus und unter Moslems; sie können ihr Leben als religiöse Wanderpilger führen, sie können auch weltlich lebende Laien sein, Männer und Frauen gleichermaßen.

3 Ich werde mich in diesem Beitrag auf religiöse Inhalte konzentrieren, die zur sogenannten Bhakti-Bewegung gehören. Mit Bhakti werden in recht unscharfer Weise die persönliche Beziehung zu einem göttlichen Wesen oder einem Absoluten bezeichnet sowie auf der sozialen Ebene die rituellen und ethischen Praktiken/Aspekte, die aus der Botschaft abgeleitet werden können. 4 Die Rolle des Guru ist nicht als auf den religiösen Bereich beschränkt, sondern globaler als Kommunikationsmedium zwischen Gruppen und Individuen zu sehen. Suthren Hirst und Zavos schlagen vor, den Guru als Begriff durch den der Meister-Schüler-Tradition zu ersetzen, um islamische wie auch säkulare Beispiele als Faktoren des Austauschs und der gegenseitigen Befruchtung ansehen zu können. "Whereas Miller is refering clearly to what he sees [speaking of the guru] as the >dynamic, sacred centre of the Hinduism`, we want to think about this idea in terms of South Asian religious traditions more generally [...]. The interaction of different teacher-pupil traditions is a significant indicator of this dynamic [...]. How might a model of teacher-pupil traditions disrupt the notion of separate world religions and give us a different way of approaching religious traditions in modern South Asia? «(Suthren Hirst und Zavos, Religious Traditions, 115-116). 
Beide Gestalten (Kabir und Sundardas) liefern Beispiele dafür, wie eine GuruGestalt entstehen kann: (1) Im Fall von Kabir gegen seine Absicht als Geschichte der Institutionalisierung einer ursprünglichen (mündlichen) Botschaft; (2) im Fall von Sundardas durch die Schriften eines Intellektuellen, durch welche die Gruppe, die sich auf seinen Guru zurückführt, konsolidiert wird und einen Bezugsrahmen erhält, der sie auf dem kompetitiven Markt und im Netzwerk der Bewegungen stärkte. Beide Beispiele sollen überprüfen, ob die Terminologie zu Gründergestalten, Überlieferern und den Verfahren zur Regulierung der Sukzession transkulturell auf die Sant-Bewegung anwendbar ist und wie religiöse Fragen zur Tradierung transkulturell problematisiert werden. Methodologisch beschränke ich mich bei der Diskussion über Sukzession auf literarische Quellen und verweise nur gelegentlich auf gesellschaftliche Aspekte, die natürlich für das Thema relevant wären, aber den Rahmen dieses Beitrags sprengen würden und mangels historischer Quellen oft nicht erforscht sind. Als religionswissenschaftliche Kategorien ${ }^{5}$ sind Begriffe wie Sukzession, Autorisierung, Legitimierung und Wissenstransfer auch auf indische Verhältnisse anwendbar, sie sollten aber nicht in ihrer westlichen Auffassung als universal gelten. ${ }^{6}$ Allerdings besteht die Gefahr, die Vielfalt Indiens zu verallgemeinern und $\mathrm{zu}$ entstellen, und die Herausforderung liegt darin, die Inhalte so gut wie möglich in ihrer Einbettung, anhand der indischen Konzepte, ${ }^{7} \mathrm{zu}$ erforschen. Indische Termini wie guru-śișya-sambhanda, sampradāya, dīkșā, paramparā haben ihre eigene interne Geschichte und verweisen auf Weltanschauungen, welche die eigentliche Schwierigkeit für Erklärungen darstellen. ${ }^{8}$ In unserem Beispiel stellt die Sant-Tradition des vormodernen nördli-

5 Ich gehe hier nicht auf die Frage der religionswissenschaftlichen Begriffsgeschichte ein. Eine parallele Begriffsgeschichte für die indischen Traditionen ist nur teilweise vorhanden, siehe Brill's Encyclopedia of Hinduism.

6 Hierzu Pollocks Aussage, dass Komparistik nicht hegemonisch sein muss und wie es sich vermeiden lässt: »But if comparison is necessary, the will to domination that sometimes seems built into the comparative method is certainly not. It is possible to produce comparison without hegemony across the human sciences. We begin to do this by making our inevitable but implicit comparison explicit, explain what role they are playing in our interpretation of our primary object, and exercise sufficient reflexivity to avoid demanding symmetry when there is only synchronicity, turning difference into deficiency, or expanding particularity into paradigm « (Pollock, »Comparison without Hegemony«, 202).

7 Auch bei diesem Aufsatz geht es letzten Endes um Wissenstransfer und dieser hängt von der Feinfühligkeit des Forschers ab, sich der Probleme der Übersetzung bewusst zu sein. Siehe Venuti, Translation Studies Reader, dort u.a. »Translation, Community, Utopia«.

8 Es gibt eine umfangreiche Wissensliteratur auf Sanskrit, die die Frage der Überlieferung erläutert und dokumentiert. Ich habe die Fragestellung absichtlich im Bereich der mündlichen und/oder Nicht-Sanskrit-Tradition platziert, um deren spezifische Merkmale zu zeigen. 
chen Indiens den Kontext dar, deren Daten die Voraussetzung für jeden Vergleich und jede Übersetzung in religionswissenschaftlichen Debatten bilden.

\section{Rahmen für einen freien Denker: die Institutionalisierung Kabirs}

Kabir ist eine relativ gut erforschte Gestalt der früh-modernen Literatur auf Alt-Hindi und es gibt reichliches Quellenmaterial für die Erforschung, wie und warum er in seiner Rezeptionsgeschichte zu einer Führergestalt oder sogar zur Gründergestalt einer neuen religiösen Bewegung geworden ist. ${ }^{9}$ Trotzdem bleiben unser Wissen spekulativ und unsere Beobachtungen vorläufig. Die Beantwortung der Grundfrage (»Wie überlebt die mündliche Lehre eines Meisters, der keine Lehre verkünden wollte? « Oder: »Kann eine unvermittelte Erfahrung, wie Kabir sie fordert, übermittelt werden?«) im Falle Kabirs hängt davon ab, ob und wie sein ursprüngliches Anliegen (seine »Botschaft«) überhaupt weitergegeben und überliefert werden kann. Wenn es sich bei dieser Botschaft um das Ergebnis einer inneren Erfahrung handelt, nämlich um das Hören des śabda (Klang und Wort) des sadguru (wahren Lehrers) als einer Art von »Offenbarung«, wie kann sie überlieferbar werden? Und welche Konsequenzen ziehen wir aus diesem Beispiel für Legitimierung und Sukzession?

Nach einigen wenigen Angaben zur Biographie (1.1) folgen Ausführungen zum Inhalt (1.2) und dem literarischen Werk, d.h. der Form und Wirkungsgeschichte (1.3), sowie eine zusammenfassende Stellungnahme (1.4).

\subsection{Biographie}

Kabir (gest. 1518) war ein sogenannter »niederkastiger« Weber aus Benares; die Hagiographie will, dass er auf dem Marktplatz saß und die Leute anredete, um sie zu einer neuen Bewusstseinserfahrung zu erwecken. Er war Moslem, wahrscheinlich aus einer konvertierten Hindu-Familie mit Verbindung zur Nāth-Bewegung, einer yoga-tantrischen Bewegung Nordindiens seiner Zeit. Kabir hat niemanden aufgefordert, sein Jünger zu werden, und hat selber keine Nachfolge organisiert oder geregelt. Lässt sich, abgesehen von seiner inneren Stimme, eine historisch

9 Zur Kabirforschung s.u. die Literatur. 
greifbare Quelle für Kabirs Wissen ausmachen ${ }^{10}$ Kabir bekennt in gewissen Versen, dass sein weltlicher Guru der berühmte Ramananda war; spätere Anhänger haben diese Genealogie ausgebaut und ihm zugeschrieben. ${ }^{11}$

\title{
1.2 Inhalt
}

\subsubsection{Gegen Autorität}

Kabirs Botschaft ist in erster Linie ein Appell an seine Hörer. Im Kontext des religiösen Establishments seiner Zeit wirkte dieser Appell provokativ. Kabir versuchte, die Menschen für ihre eigenen Erfahrungen aufgeschlossen und bewusst zu machen; deshalb rief er sie dazu auf, nicht blind den Anweisungen hypokritischer Autoritäten wie Pandits (hinduistische Gelehrte) und Mullas (muslimische Gelehrte $)^{12} \mathrm{zu}$ folgen. Man sollte nur direkt frisch von der Quelle lernen und nicht aus Büchern oder von den sogenannten Gelehrten.

Du beachtest den Ramadan, betest zu Allah, aber schlachtest Lebewesen aus Gelüst.

Du siehst nur dich, nicht die anderen, womit verlierst du deine Zeit.

Kāji, in dir ist der Herr eins, dein Denken ist schwach und du siehst nicht. [...]

Allah ist versteckt in jedem Körper, verstehe dies mit dem Herzen.

In beiden, dem Hindu und Türken, gibt es nur das Eine, so sagt Kabir. ${ }^{13}$

Er forderte seine Hörer und Gefolgsleute auf, keinem Guru und keiner Belehrung zu folgen, die von Brahmanen oder Priestern kontrolliert wurde:

\author{
Was nützt Baden und Waschen, \\ Wenn du im Inneren nicht Rāma gefunden hast? \\ Was nützt es, den Körper äußerlich zu reinigen, wenn Innen überall Schmutz ist? [...] \\ Du quakst wie Frösche im Gangeswasser. Ohne Hari gibt es keine Erlösung. ${ }^{14}$ \\ Sie erzählen heilige Geschichten, sie stehen früh auf, um Lügen zu sagen. [... $]^{15}$
}

$10 \mathrm{Zu}$ historischen Quellen für seine Lehren, siehe Burger, »Kabīr«.

11 Die Hagiographie von Anantadas greift die in der Überlieferung akzeptierte Idee auf, dass Kabir, obwohl er Moslem war, durch einen Trick von Ramananda initiiert wurde: Kabir soll sich Ramananda in den Weg gelegt haben, als dieser zu seinem Morgenbad ging. Als Ramananda über ihn stolperte, sagte er »rāma« (etwa »o Gott!«); mit diesem heiligen Rāma-Mantra initiierte er Kabir unabsichtlich in seine Tradition (sampradāya). Lorenzen, Kabir Legends.

12 Mulla ist die südasiatische Bezeichnung für »Meister (Gelehrter) (arab. mawlah).

13 Rāgū àsā 29 (Vaudeville, Kabìr-Vāṇī, 333).

14 Kyā hvai 346 (Vaudeville, Kabìr-Vānī, 207).

15 Ramainī 61 (Singh, Kabīr Bïjak, 100). 
Insofern der soziale Status (wie die Zugehörigkeit zum Brahmanenstand) erblich ist, lehnte er Kastenunterschiede als Bedingung für Zugang zu Religion ebenso wie erbliche religiöse Funktionen ab, da sie nicht auf religiöser Erfahrung beruhen. ${ }^{16}$ Indem er alles ablehnte, was nicht durch die eigene Authentizität (Erfahrung) belegt wird, verkündete Kabir eine provokative Botschaft, weil er damit die Anhänger jeglicher Glaubensrichtung der blinden Gefolgschaft anklagte.

\subsubsection{Umgangssprache}

Aus der Ablehnung der personifizierten religiösen Autoritäten ergibt sich, dass er den Anspruch, dass nur Sanskrit die Sprache zum Ausdruck spiritueller Gefühle sei, ablehnte zugunsten der lokalen Umgangssprachen. Kabir, sicher einer der Stifter dieser anti-brahmanischen Einstellung der Sants, besteht darauf, dass sie das Sanskrit und das Erbe auf Sanskrit abgefasster Texte als unwichtig (oder sogar als Hindernis) für den religiösen Weg betrachten und direkte Erfahrung vorziehen, die sich dann in den jeweiligen lokalen Idiomen ausdrückt. Die Umgangssprache wird als sprudelnde Quelle gesehen, das Sanskrit als das trübe Wasser des Teiches. ${ }^{17}$

\subsubsection{Sadguru/(Gottes-)Erfahrung}

Der Appellcharakter von Kabirs Botschaft ergibt sich daraus, dass sie Zeugnis für eine religiöse Erfahrung ist. Er forderte auf, sich ausschließlich auf die eigene Erfahrung zu verlassen, wie sie sich im Inneren, im Herzen oder im Gemüt offenbart; Kabir nennt diese Instanz von Religion und Spiritualität den »wahren Guru« (sadguru). Die Erfahrung ersetzt die Belehrung durch den Guru und ist also der eigentliche Lehrer.

Kabir, der Geist ist eine Biene geworden, und hat eine ewige Stätte gefunden.

Der Lotus, der ohne Wasser blüht, nur dienstbare Nahestehende können dies sehen. ${ }^{18}$

16 So wie das schon genannte Merkmal, dass weder Kaste noch kastenspezifische Reinheitsvorschriften eine Rolle spielen, so wenig wie Opferkult oder komplexe Rituale.

17 Wichtig ist, dass die Sant-Bewegung dazu beigetragen hat, die Umgangssprachen zu Bildungssprachen zu machen.

18 Kabīr Granthāvalī 6 (Vaudeville, Kabīr Granthāvalī, 13). 
Der wahre Guru ist der Meister, der zu jenen spricht, die in ihrem Leben ausschließlich dem inneren Guru gehorchen und seine Macht und Schönheit preisen.

Im Körper ist das Unerreichbare erfasst, im Unerreichbaren ein Weg.

Sagt Kabir, ich habe die Erfahrung erlangt, der Guru hat den Weg gezeigt. ${ }^{19}$

Für Kabir muss der Mensch sich völlig dem sadguru unterwerfen, in totaler Unterordnung und ohne eine Spur von Ego. Dies wird - nicht zuletzt durch die Terminologie, mit der Appell und Erfahrung formuliert werden - zu einem Merkmal eines Sant.

Jene, die dem Stolz der Abstammung entsagt haben und die Erlösung suchen, indem sie den Sprössling und den Samen vernichteten, erreichen den Zustand der Lebend-Erlösten. ${ }^{20}$

Die Beziehung zum sadguru kann manchmal eine Liebesgeschichte werden; er selber vergleicht seine Gefühle mit jenen von Liebenden; oder sie kann als der individualistisch-einsame Weg yogischer Verwirklichung gesehen werden. Für den Sant Kabir kann die Beziehung weitergegeben werden, aber nicht durch Licht, Berührung oder Regeln (alles Elemente, die in der Bhaktibewegung existieren), sondern in seinem Fall durch Selbstverwirklichung, wie sie durch das Hören auf den einzigen wahren Meister im Inneren jeder Person oder durch das Zusammensitzen mit jenen, die sich derselben Suche verpflichtet fühlen, eintritt.

Ich habe viele muslimische Meister und Heilige getroffen;

Sie studieren das heilige Buch;

Sie lehren ihre Schüler viele Heilsmittel,

(Aber) wie steht es um dieses Wissen in ihnen? ${ }^{21}$

Als Autorität und Vorbild anerkannt wird jemand, der Gott erfahren und verwirklicht hat, statt nur darüber zu reden. Diese Autorität muss für die Nachfolger erkennbar sein. Es werden diese Führer, die jenes »Etwas« verwirklicht haben, das ihnen Autorität in religiösen Angelegenheiten verleiht, mit verschiedenen Namen bezeichnet, die teilweise das abdecken, was wir Charisma ${ }^{22}$ nennen: jivvanmukta bedeutet »lebend befreit«, guru bezeichnet den Meister, bhagavān eine

19 Kabīr Granthāvalī 9 (Vaudeville, Kabīr Granthāvalī, 13).

20 Ramainī 35, übersetzt nach Vaudeville, Au cabaret de l'amour, 57.

21 Śabda 4 (Singh, Kabìr Bījak, 111).

22 Ich beziehe mich hier auf den Begriff, wie er von Max Weber in seinem grundlegenden Werk Wirtschaft und Gesellschaft über religiöse Autorität und die Konstruktion religiöser Tradition von der Gründergestalt zur Institutionalisierung verwendet wird. 
heilige, von göttlicher Gnade erfüllte Person, jogī ist der Wanderasket, avatār die Bezeichnung für eine göttliche Inkarnation usw. Diese Namen erläutern Aspekte der befreiten wahren (sant) Person.

Gotteserfahrung ist also Gottesbeziehung; und Beziehung zum Guru (zum wahren, inneren Guru) fällt damit zusammen. Das Hören des śabda (Klang und Wort) des sadguru (wahren Lehrers) ist eine Art von »Offenbarung«. Jeder solle sich auf seine eigene Erfahrung verlassen; Kabir wies lediglich auf die Fähigkeit jedes einzelnen hin, diesen wahren Lehrer im Inneren selber zu entdecken.

Der Mittelpunkt seiner Botschaft war der Abgrund der Liebe zum Absoluten und das Vertrauen in die Güte des Schöpfers der Welt, denen das Ego sich widersetzt.

Solange man sagt: das gehört mir,

Solange kann nichts geschehen.

Wenn man verzichtet zu sagen: mir, mir,

Da kommt der Herr und erfüllt unsere Wünsche.

Denke über diese Weisheit nach:

Warum rufst du nicht Hari an, der vom Leiden befreit. ${ }^{23}$

Oft mit einem persönlichen Namen versehen (Rāma, Hari), bleibt jedoch das Geheimnis des Absoluten ein Hauptteil seiner Auffassung des Göttlichen.

Als es weder Wind noch Wasser gab; wer hat dann die Welt erschaffen.

Als es weder Knospe noch Blume gab, weder Schoss noch Samen.

Es gab weder Wissenschaft noch Veda, kein Wort, keinen Retter [...]

Es gab weder Guru noch Schüler, weder Weg noch Nichtweg, keine zwei Straßen;

Wie das Unkennbare benennen, das weder Dorf noch Ort hat?

Wie soll ich das nennen, das Attribute hat und nicht hat. ${ }^{24}$

\subsubsection{Praxis}

Hören. Ob das Hören der inneren Stimme (śabda) als etwas verstanden wurde, das man einüben und regelmäßig praktizieren soll, ist nicht deutlich. Ob Kabir selber davon ausging, dass das Singen und Hören seiner Liedgedichte eine spezifische religiöse Praxis sei oder sein sollte (vergleichbar dem Murmeln heiliger Silben oder Mantras im Yoga und in der Bhaktipraxis), ist ebenfalls fraglich; für

23 Bhairau 14, übersetzt nach Vaudeville, Au cabaret de l'amour, 128.

24 Ramainī 7 (Singh, Kabìr Bïjak, 82). 
seine Nachfolger dagegen lässt sich diese Praxis bestätigen. Übungen zum Hören der inneren Stimme sollen zur Befreiung und zur Vermeidung von Leiden, Tod und Wiedergeburt führen.

Meditation. Das Wort (śabda) bezeichnet den Mittelpunkt als innere »Offenbarung«, die direkt vom »Göttlichen« ausgeht. Indem sie über śabda meditieren, bemühen Sants sich um die Verschmelzung mit dem Geistprinzip, um dadurch die Befreiung zu finden. Dazu hilft auch die Rezitation des Namen Rāmas (japna), die ununterbrochen die Stille des Geistes herbeiführt. Auf dieser Ebene ist der Einfluss der nāthayogī sehr deutlich, wie sie soll die Rezitation zur Nichtrezitation führen (ajapnajapna), d.h. der Rezitierende muss so weit kommen, dass seine Rezitation sein Atem wird.

Satsanga, kīrtana. Sants haben als hauptsächliche Praktiken Zusammenkünfte für gemeinsames Singen (kïrtana), was als Ausdruck der Verehrung des göttlichen Namens (śabda) verstanden wird. Durch das gemeinsame Singen der Liedgedichte wird die durch sie übermittelte Erfahrung zu einer Gemeinschaftserfahrung, zumindest theoretisch. Gemeinschaft ist Voraussetzung jeglicher Kommunikation und also jeglicher Sukzession. Und sie ist vielleicht ein Beleg dafür, dass Erfahrungen, wie Kabir sie für sich beansprucht, de facto nicht häufig auftreten: Wer sie gehabt hat, hat keine Gemeinschaft mehr nötig; der Lebenderlöste hat die Gesellschaft hinter sich gelassen.

\subsection{Werk}

\subsubsection{Liedgedichte}

Die Überlieferung berichtet, dass Kabir Verse verfasste, oft in einer ungehobelten, gelegentlich hermetischen Sprache, die gesungen wurden. ${ }^{25}$ Er komponierte und sang Verse, bekannt als pada und dohā, die beide metrische Formen waren, um religiöse Ideen zu verbreiten. Dohās werden auch sākhīs (»Zeugnis«) genannt, wurden mit Melodien in vordefinierten rāgas gesungen und geben Zeugnis über Lebenserfahrungen, meistens in zwei sich reimenden Linien gleicher Länge. Padas haben eine lockere metrische Struktur und stehen volkstümlichen Traditionen näher. Die eher hermetischen Aussagen stehen der Sprache der Sadhus,

25 Zur literarischen Form, siehe Hess, »Three Kabir Collections«. 
auch saddhukkarībhāṣā (»Wanderasketensprache«) genannt, nahe und sind oft erklärungsbedürftig.

\subsubsection{Mündliche Verbreitung, Musikalität}

Anfänglich wurden seine Liedgedichte mündlich vorgetragen und dadurch weitergegeben und von fahrenden Sängern, Händlern, Reisenden (Pilgern), Jogis über weite Teile Nordindiens verbreitet. Während die Sänger die Liedgedichte Kabirs verbreiteten, konnten sie selber Lieder verfassen und Kabir zuschreiben, indem sie in eigenen Worten oder in der Diktion der Gegend, in der sie vorsangen und komponierten, Kabirgedichten nachempfundene Texte schufen. Daher ist es sehr schwierig zu entscheiden, welche Verse wirklich Kabir als Autor haben. Seine Botschaft wurde über das Singen von Liedgedichten verbreitet, durch die Mitteilung poetischer Kompositionen und durch die Kraft der Lehren und der Überzeugung, durch die die Menschen neue religiöse Ansichten annahmen. Seine Botschaft fand Gefallen und wurde über Generationen hin weitergegeben. ${ }^{26}$

Singen ist eine Art der Weitergabe, die ein besonderes Gedächtnis verlangt. Erstens wird die Sprache an die Erfordernisse des Reims angepasst, aber dann beeinflussen Rhythmus und melodische Strukturen das Gedicht und seine Bedeutung. ${ }^{27}$ Beim Singen, zweitens, kann der Dichter ein Wort hervorheben, indem er es länger oder wiederholt singt, um eine besondere Wirkung zu erzeugen. Das bedeutet, dass die Ausführung, der performative Aspekt, ein Schlüsselelement für Gedächtnis und Überlieferung ist. $\mathrm{Zu}$ den Strukturmerkmalen der indischen Musik bzw. Aufführungspraxis gehört es, dass über bzw. mit einem definierten Repertoire ausgewählter Noten improvisiert wird, ohne feste Melodie und ohne festgelegte Takt- oder Rhythmusabfolge.

Die Aufführungspraxis wird auch der Ausgangspunkt für die Varianten (»Lesarten«) sein, die von einem Gedicht existieren und die in den verschiedenen Strängen der Handschriftenüberlieferung übernommen wurden und erhalten sind. Die mündliche und performative Überlieferung, welche die Struktur der Gedichte ebenso wie ihren Sinn stark prägt, kennzeichnet die Überlieferung eines Erbes, dessen Anfang offen und flexibel ist und nicht an eine Person oder an einen Autor oder Gründer gebunden ist. Das soll nicht heißen, Kabir hätte

26 Die vollständige Geschichte von Kabirs Erbe muss erst noch geschrieben werden; siehe Lorenzen.

27 Auch wenn es sich um einen anderen Dichter, Surdas, handelt, ist die Erklärung Ken Bryants zur Beziehung zur Musik aufschlussreich (Bryant, »Introduction«). 
nicht existiert, aber seine Gedichte stammen von ihm und seinen Nachdichtern und bilden so eine Kabirtradition. ${ }^{28}$

Unter den Tausenden von Versen Kabirs, die in den heutigen Sammlungen (Granthāvalī) enthalten sind, wurden nur die wenigsten von Kabir selber gesungen. Darin besteht von Anfang an ein wichtiges Kennzeichen von Sukzession und Autorschaft.

\subsubsection{Verschriftlichung und Institutionalisierung}

Die Gedichte, welche die Form dieser Erfahrung und Authentizität waren, haben eine lange Wirkungsgeschichte. ${ }^{29}$ Als die Menge der Liedgedichte, die Kabir zugeschrieben wurden, anwuchs, verwendeten die Sänger Aufzeichnungen als Gedächtnisstütze. Dies waren die Vorläufer der Sammelhandschriften, d.h. jener Manuskripte, die ab dem 17. Jahrhundert von diesen Liedern gemacht wurden.

Seine Botschaft wurde jedoch auch sehr rasch den Zwängen einer »Schule» (panth, wörtlich »Weg«) unterworfen. Die Sukzession spielt sich dann im Rahmen eines Ordens ab, ebenfalls sampradāya oder panth genannt, ${ }^{30}$ wobei eine eher soziale Organisation gemeint ist, innerhalb welcher die Überlieferung gestaltet und vor allem verändert wird. Über die ersten Jahrzehnte nach dem Tod Kabirs und die Entwicklung einer Schule (eines »Weges«) gibt es keine Informationen, auch wenn die hagiographischen Angaben über die Nachfolger diese als direkte Jünger darstellen und somit von einer ununterbrochenen Genealogie ausgehen. Historisch gesprochen wird der Weg als Schulrichtung erst im 17. Jahrhundert zum ersten Mal erwähnt, auch wenn er schon vorher in lockerer Form bestand. ${ }^{31}$ Institutionalisierung umfasste die Gründung von Klöstern, den Erwerb von Besitz, die Aufteilung religiöser Funktionen, die Verschriftlichung der mündlichen Überlieferung und die Einrichtung von Ritualen.

28 Was dann später als Kabirpanth, d.h. »Kabir-Weg« bezeichnet wird. Panth ist ein anderer Name für sampradāya (siehe Anm. 30).

29 Heutzutage kennt man drei Hauptrezensionen: Kabīr Granthāvalī, Bïjak, Adīgranth.

30 Sampradāya ist abgeleitet von der Verbalwurzel dā mit den Präpositionen sam- und pra- und heißt wörtlich "weitergeben, übergeben«, entspricht also etymologisch recht genau unserem »Tra-di-tion«. »The word sampradāya comprises the process as well as the result. It is stradition as transmitted , which means that the transmission process is the basis of the authority and the validity of tradition « (Malinar, "Sampradāya«). Die Nachfolger Kabirs teilen diese Auffassung, wobei Kabir selber eher einen Bruch dieser Vision bedeutet.

31 Burger, »Kabīrpanthīs«; Bahuguna, »Conflict and Assimilation«. 
In den heutigen Kabirüberlieferungen sind zwei Stränge zu unterscheiden. Einerseits gibt es viele Anhänger ohne Initiation, welche Kabirs Ansichten als freie Denker außerhalb von Institutionen teilen. Andererseits gibt es die Angehörigen des Schulweges (panth), die sogenannten Kabirpanthis, welche initiierte Jünger sind und sich im Laufe der Zeit in viele Zweige und Unterzweige gespalten haben. Aus dem losen Zusammenschluss einer Anhängerschaft, welche Moslems und Hindus gleichermaßen einschloss, wurde der panth immer hinduistischer und organisierter im Einklang mit dem Mainstream-Hinduismus. Die zwei bekannten Zweige von Benares und Chattisgarh zeugen für den institutionalisierten Charakter des panth; sie praktizieren zwei Arten der Sukzession, eine erblich, die andere durch Wahl. Beide halten am Prinzip der Initiation fest. ${ }^{32}$

Der Zweig in Benares (das Hauptquartier bzw. das »Ursprungskloster«, mūlamațha) und der andere in Chattisgarh haben sich bei den Institutionalisierungsprozessen dem Vorbild der verbreiteten vishnuitischen Sekten angepasst. ${ }^{33}$ In dem Zweig von Benares wird der Führer des Klosters ācārya (eigentlich »Lehrer«) genannt, und er hat begonnen, auf Sanskrit zu veröffentlichen. In Chattisgarh hingegen beruht die Sukzession des Guru auf Erblichkeit. Einige seit Kabir bekannte Merkmale wie die Ablehnung eines (personifizierten) Gottes werden aufrecht erhalten, andererseits ist zum Beispiel das Kasten- oder Reinheitsbewusstsein in die Schule von Benares zurückgekehrt, wo man nicht gemeinsam isst. Wie so oft in der indischen Geschichte versucht man, seine Auffassungen dem brahmanischen Ritualleben anzupassen. ${ }^{34}$

\subsection{Auswertung}

Die Kabir zugeschriebenen Liedgedichte sind sicher ein ausgezeichnetes Beispiel, um zu illustrieren, wie Spiritualität oder Religion in diesem Kontext funktionieren. Man hört oft sagen, wie ungewöhnlich Kabir sei, aber dies unterstellt eine falsche Sichtweise, da es viele Sänger und Dichter gab, die Kabirs Namen verwendeten, um auszudrücken, dass sie mit seiner Denkweise einverstanden waren. Wenn jemand im Geiste Kabirs singt und den Namen Kabirs am Ende des Liedes

32 Aufschlussreich ist, dass die meisten der heute sehr populären Liedverse Kabirs nicht in den ältesten Handschriften belegt sind; dies bekräftigt, dass es sich um eine lebende Tradition handelt und dass die Kriterien der Authentizität und Urheberschaft/Autorität in diesem Fall nicht anwendbar sind. Selbst in den institutionalisierten panths wird die Akzeptanz der Texte sehr locker und ohne Rücksicht auf die frühen Handschriften gehandhabt.

33 Burger, »Kabīrpanthīs«; Lorenzen, »Kabir-Panth«; ders., »Dissent in Kabir«.

34 Die Rezeptionsgeschichte Kabirs in Pakistan wird hier nicht behandelt. 
einfügt, dann wird das Gedicht als ein Lied Kabirs und der Kabirtradition anerkannt. Somit ist Autorschaft nicht an eine einzelne Person gebunden, sondern an eine Gruppe von Menschen mit ähnlicher Geisteshaltung. Natürlich hat die historische Forschung versucht, die »echten « Kabirtexte zu identifizieren - ohne überzeugenden Erfolg, auch wenn dieser wichtige Arbeitsaufwand zu vielen Erkenntnissen und kritischem Denken geführt hat.

Wie wurde Kabirs Erbe so erfolgreich? Denn man kann wirklich von einer Erfolgsgeschichte sprechen, die bis heute weitergeht und verschiedene politische, wirtschaftliche und spirituelle Programme und Zielsetzungen umfasst. Zusammenfassend lässt sich das Erbe von Sant Kabir als ein typischer Fall der Wende von einem freien Denker zu einer Institution bezeichnen, wobei gesellschaftliche, politische und religiöse Zwänge diese Wende erklären, die den panth einer Sichtweise eingegliedert haben, die man - faute de mieux - »brahmanisch « nennen muss.

Institutionalisierung ist vielleicht möglich für Verfahren zur Erlangung göttlicher Gnade, aber sicherlich nicht für die von Kabir verkörperte Errungenschaft. Es ist nicht bekannt, dass Kabir seine innere Erfahrung direkt durch Besessenheit oder Übertragung yogischer Kräfte weitergegeben hätte. Es scheinen vielmehr die Liedgedichte die Träger seiner Botschaft von einer Zugänglichkeit religiöser Erfahrung für alle gewesen zu sein und somit die Zeugen und Auslöser für die Botschaft. Kabir war kein Guru im üblichen Sinn und er wollte keiner sein. Seine Jünger allerdings machten ihn zu einer Figur, deren Botschaft es sich zu folgen lohnte. Dass Kabir existierte und eine revolutionäre Botschaft verkörperte, steht außer Zweifel; aber er war keine Gurugestalt und die Überlieferung muss in seinem Fall als Medium für eine allgemeine Erweckung zu Möglichkeiten neuen religiösen Bewusstseins für verschiedene Bevölkerungsschichten des indischen Subkontinents betrachtet werden.

Vom Gesichtspunkt einer Religionswissenschaft aus, die an Sukzession interessiert ist, bleibt zu klären, wie die innere Erfahrung des śabda übermittelbar werden kann. Die Auswertung der Rezeptionsgeschichte Kabirs in den panths scheint darauf hinzuweisen, dass der Mittelpunkt von Kabirs Botschaft nicht übermittelbar war und dass das, was erhalten wurde, vorwiegend äußerliche Strukturen waren, die im Lauf der Zeit immer dominierender wurden und die dem Vorbild der wichtigen Sekten, zum Beispiel der Vishnuiten, folgten. Und das sagt etwas über »Religion« und könnte also Konsequenzen für die akademische Disziplin haben, die »Religion« erforscht, die Religionswissenschaft eben, in deren Rahmen wir über Sukzession nachdenken. Von den Sants als einer (hinduistischen, indischen) »Religion « zu sprechen und von den Zweigen als deren »Sek- 
ten «, ${ }^{35}$ akzentuiert markant, wie verfehlt und unbefriedigend die Übertragung von Begriffen sein kann. »Weg«, »Bewegung «, "Strömung«, »Zweig« sind auch nur metaphorische Annäherungen, aber eben welche, die indischen Ursprungs sind (panth, mārga, paramparā,${ }^{36}$ sampradāya, śākha ...) und in westliche Redeweise übertragen werden.

\section{Sundardas (1596-1689): Erhalter einer Tradition}

Sukzession im Falle Kabirs dem Beispiel von Sundardas gegenüberzustellen, schlägt eine Brücke zu einer anderen Epoche in der Geschichte der Santbewegung und zu einem anderen Zweig innerhalb dieser Bewegung. Auch ihr Gründer, Dadu bzw. Sant Dādū Dāyāl aus Rajasthan, ${ }^{37}$ wird als Sant betrachtet; und der Ausdruck panth (»Weg, Schule«) wird auch auf die Überlieferung (paramparā) angewandt, die er gezielt und bewusst aufbauen wollte. Zur Zeit des Sundardas waren dies feste Kategorien für die Überlieferungsrichtung, für den panth, zu dem er gehörte. Sundardas ist ein Autor der zweiten Generation im Kontext eines bereits gefestigten panth, welche auf eine Gründergestalt zurückgeht. Sein Wirken dokumentiert, wie ein Gelehrter in der frühen Geschichte des Dadupanth die Stellung dieser Schule auf dem religiösen Markt durch seine Schriften stärkte. Schrifttum anstelle von mündlicher Überlieferung war die treibende Kraft, welche das Profil dieser Schule von Anfang an definierte.

In diesem Fall ist die Initiation (dīkșā) der Schlüssel für die Gewährleistung der Kontinuität des panth von Anfang an. ${ }^{38}$ Dadus Nachfolger Raidas sowie andere unmittelbare Gefolgsleute der Bewegung legten großen Wert darauf, die Sukzession sicherzustellen. ${ }^{39}$ Raidas initiierte Gefolgsleute und schuf somit einen panth mit (eigener) Tradition.

35 Siehe Malinar, »Samprādaya«, zu Sekte.

36 Paramparā zeigt noch mehr den Schulaspekt und wird verstanden als die Sukzession von Meister zu Schüler, oft im Nachhinein erschlossen. Man spricht auch von guruparamparā, im religiösen Kontext wie bei der Kunst.

37 Zum Dadupanth siehe Thiel-Horstmann, Crossing the Ocean.

38 Die Initiation liefert dann Merkmale, die die Gruppe identifizierbar machen, wie z.B. ein Mantra, oder Merkmale von Zeichen und Kleider. Auch die initiierten Kabirpanthis haben ihre erkennbaren Merkmale.

39 Wir wissen darüber aus der Biographie Jangopāls, eines direkten Jüngers von Dadu. 
Dadu ist ein Gründerguru, der seine Form der Sant-Spiritualität als einen »mittleren Weg« vertritt, weniger radikal als die polemischeren Formen einer Santbewegung wie die von Kabir.

\footnotetext{
Welchen anderen Heiligen es auch geben mag, ich ehre sie alle. Aber über Guru Dādūs Ruhm will ich sprechen.

Die wundervollen Ereignisse lassen sich nicht in Worte fassen. Welche Zunge hätte dies je vermocht? ${ }^{40}$
}

Dadu selber hatte keinen menschlichen Guru, aber er hatte zweimal in seinem Leben die Vision des mystischen Guru (wie der innere Guru bei Kabir). Im Gegensatz zu Kabir wollte Dadu das Weiterleben seiner Ansichten sichern. Es wird berichtet, dass er einige der Handschriften, in denen seine Aussprüche aufgeschrieben wurden, selber eingesehen (und also vielleicht geprüft) hat. Sein Schüler Bakhna ist der erste, der die Nachfolger Dadus »Dadupanthis «nennt; er zählt 52 Jünger der ersten Stunde auf. ${ }^{41}$ Ein halbes Jahrhundert später haben wir bereits einen panth, aber wir wissen, dass dieser trotzdem noch umstritten war.

Auseinandersetzungen über die Sukzession sind Teil der frühen Geschichte des panth. Bezeichnenderweise spricht die erste Generation der Nachfolger über Dadu, während die zweite Generation ihn nicht mehr erwähnt. ${ }^{42}$ In späteren Generationen taucht er als der anerkannte Gründer der »Sekte« wieder auf. Die idealisierte Gestalt Dadus einerseits, Handschriften andererseits und die Gründung heiliger Orte tragen zur Konsolidierung des panth bei. Der Ort Narain in Rajasthan wird ab dem 18. Jahrhundert zum Hauptquartier des panth; der Abt dieses Klosters sollte ein Brahmane (durch Geburt) sein. Er wird ācārya genannt, was ein an eine Funktion geknüpfter Titel ist. Ein Guru wird nicht gewählt.

Welche Rolle spielte also Sundardas in der Sukzession des panth? Sundardas traf Dadu, als er sieben Jahre alt war, aber er wurde erst später initiiert. Nach seiner Initiation wurde Sundardas nach Benares geschickt, um eine formale Ausbildung in traditionellen Wissenssystemen zu erhalten. Wir wissen nicht,

40 Jangopāl, Dādūjanmalīlāparacī 1,4 (Thiel-Horstmann, Crossing the Ocean, 110).

41 Auch diese Gemeinschaft war vom Vorbild der vishnuitischen Sekten beeinflusst und benutzte sie als Modell, um auf vier nirguna-Sekten (Sekten, die durch einem Begriff des »Absoluten ohne Eigenschaften« gekennzeichnet waren), zu kommen: Nanak, Kabir, Dadu, Niranjani mahant Jagannath. Der Bhaktamāla, ein wichtiger Text, der über die Sekten des 16. bis 17. Jahrhunderts spricht, ordnet in der Tat den (Dadu-)Panth parallel zu den vier orthodoxen vishnuitischen Sekten ein. Dadurch erhält man vier nirguna-Sekten. Thiel-Horstmann, Crossing the Ocean, 1-20.

42 Siehe Thiel-Horstmann, Crossing the Ocean. 
mit welchem Titel er angeredet wurde und was seine Stellung innerhalb des panth war. Wir wissen jedoch, dass er Abhandlungen schrieb, in denen er Dadus Ansichten und seine eigenen erklärte. Er war daher eher ein ācārya, hier im Sinne eines gelehrten Lehrers. Er ist weder eine Gründergestalt noch ein Guru. Wie Kabir wurde auch Sundardas Sant genannt, da die »Schule«, zu der er gehört, als eine Richtung der Sant-Tradition angesehen wird. Er verwendete Strategien zur Stärkung der Tradition, obwohl aus seinen Äußerungen offensichtlich ist, dass er nicht mit Dadus Lehren in Einklang war. Es ist nicht klar, ob dies eine bewusst gewählte Strategie des Sundardas war. Für ihn waren der panth und der Guru die wichtigen Größen, an denen festgehalten werden musste, aber der begriffliche, dogmatische Inhalt der Lehre war nicht entscheidend. Ihm lag wenig daran, mit dem Guru einer Meinung zu sein, obwohl er ihn in seiner Funktion als Gründer voll akzeptierte und pries. Sundardas scheint verstanden $\mathrm{zu}$ haben, dass es für das Überleben und die Zunahme der Wichtigkeit des panth unerlässlich war, den Anschluss an den anerkannten Mainstream zu finden. Die Art und Weise der Weitergabe war für ihn allerdings nicht die mündliche Tradition, sondern die Schriftlichkeit, die als selbstverständlich die Hochschätzung des Meisters voraussetzte. Inwiefern die Schriftlichkeit sich aus Hochschätzung des Meisters ergibt, ist fraglich, scheint aber für Sundardas unproblematisch gewesen zu sein.

Sundardas schrieb viele Würdigungen und Hymnen, die die Erhabenheit seines Meisters Dadu Dayal besingen, ohne welchen nichts möglich ist, der der Grund von allem ist.

Denn Guru Dādū ist gekommen; er hat bewirkt, dass das Wort gehört wurde; er hat vom Ewigen Höchsten Selbst gesprochen. ${ }^{43}$

Sundardas, der Dichter und Theologe der zweiten Generation im panth, sieht den Guru in erster Linie als vollkommenen Lehrer. Er ist mehr als ācārya (Lehrer im Sinn von Ausbilder), er ist der Guru, der einen verwandelt und somit spirituell überhaupt erst erzeugt. Trotz dogmatischer Unterschiede nimmt Sundardas Dadu als den vollkommenen Guru an, obwohl er Dadus Ansichten nicht wirklich teilt. Sundardas ist eigentlich ein Advaitin, ein Nicht-Dualist, der jedoch - anders als die Māyāvadīns (die Anhänger der Lehre, dass die Welt ein auf irrtümlicher Erkenntnis beruhender Schein ist) - an die Realität der Welt glaubt. Den Meister zu preisen, ist eine Konvention, um zum panth zu gehören, aber er wirkt in seinen Schriften darauf hin, dass der eigene panth im weiteren Feld religiöser Traditio-

43 Gurukṛpā-așțaka (1) (Thiel-Horstman, Crossing the Ocean, 142). 
nen anerkannt wird. ${ }^{44}$ Dies ist analog zur Rolle Kabirs. Die Funktion des Guru, Erfahrung auszulösen, begründet, dass die Lehre und das Schriftentum, welches Lehren enthält, vom Guru unabhängig und geändert werden können, ohne den Guru zu wechseln oder die Hochachtung für ihn zu verlieren. »Lehren « sind von der Erfahrung unabhängig. So ist Dadu unangreifbar und hat das Höchste realisiert, mehr als jene, die sich mit der Seele, mit Gott verbinden.

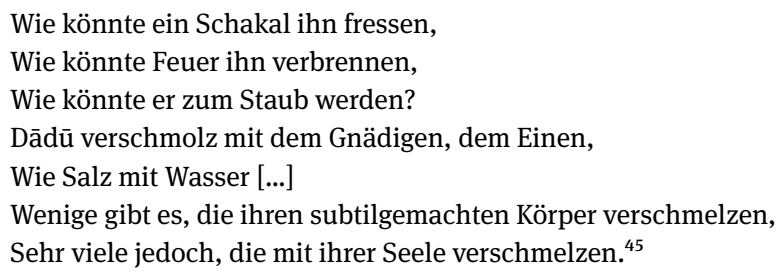

Sundardas ist ein hochbegabter und angesehener Dichter und versteht sich auf den Umgang mit dem Wort und seiner Macht. Sein Bemühen, systematische Abhandlungen zu verfassen, lieferte dem panth Schriften, deren Wert darin bestand, dass sie vorgezeigt werden konnten. Sundardas setzt die Tradition an die Stelle des Guru. Statt die Tradition abzuschaffen, wird sie zum notwendigen Rückgrat von Religion. Der Meister wird vor allem nur als Garant des richtigen Verständnisses der Tradition angerufen. Zur Zeit von Sundardas wurden sogenannte sarvangīs geschrieben, d.h. einzelne Überlieferungstränge übergreifende, synthetisierende Textsammlungen, welche einen faszinierenden Beleg für das (Text-)Gedächtnis des 17. Jahrhunderts darstellen. Er selber folgt dieser literarischen Konvention. Als Beispiel für eine systematisierende Abhandlung sei auf seine Zusammenfassung der Yogaüberlieferung in dem Sarvānga yoga pradīpaka ${ }^{46}$ genannten Text verwiesen. Sundardas will darin alle Traditionen, die von Yoga handeln, berück-

44 Darin steht er dem Sikh panth nahe; die Zusammenstellung des Adīgranth wurde etwa 1605 begonnen und sie bildete das starke Fundament des Sikh panth. Dies könnte jemandem wie Sundardas, der gebildet und belesen war, als Vorbild gedient haben. Die panths ahmten die Strategien anderer panths nach; der Sikh panth verdient, als starkes Modell angeführt zu werden. Die von Arjun Singh, dem 5. Guru der Sikh-Tradition, hergestellte Kompilation von Texten (stipuliert um 1605) markiert ein Wahrzeichen im Prozess der Schaffung von Kennzeichen für die Anhänger dieser Bewegung. Dies kristallisierte sich weiter heraus mit der Formalisierung des panth unter dem 10. Guru als einem Khalsa Panth (Initiation, Definition, Identifikationsmerkmale usw.). Warum der panth des Sikhismus als Weltreligion anerkannt wird, viele andere, wie die Kabirpanthis, dagegen nicht, bleibt eine offene Frage.

45 Jangopāl, Dādūjanmalīlāparacī 79, übersetzt nach Thiel-Horstmann, Crossing the Ocean, 118. 46 Siehe Burger, »Sarvānga yoga pradīpaka«. 
sichtigen. Es ist fast das Werk eines Enzyklopädisten, hätte er nicht den Ehrgeiz, seinen eigenen Advaita-Yoga als den besten Weg darzulegen. Trotz der Neigung zu deskriptiven und allgemeinen Darlegungen ist sein Wissen deutlich auf seine eigenen Überzeugungen hin orientiert. Als Strategie impliziert dies, dass er dem eigenen panth einen umfassenden Überblick über andere Traditionen liefert und die eigene Tradition dadurch dem weiteren Feld der Sanskritüberlieferung zuordnet, ohne den Anspruch auf die Besonderheit und Überlegenheit der eigenen Überzeugungen aufzugeben.

\section{Schlussbemerkungen}

Es könnte sein, dass die Terminologie, die in der Religionswissenschaft zum Beispiel auf Englisch oder Deutsch für die Rolle und die Funktionen des Guru und deren Sukzession und Überlieferung verwendet wird, die Sachverhalte der SantBewegung nicht hinreichend treffend wiedergibt, so dass sich, einmal mehr, die Frage nach der Übersetzbarkeit der indigenen Begriffe stellt und mit ihr die - für den Schritt hin zu einer globalen Religionsgeschichte unerlässliche - Aufgabe, eben dies zu thematisieren. Innerhalb der Sant-Bewegung finden wir grundsätzlich unterschiedliche Merkmale und Strategien der Sukzession, die den einheitlichen Begriff eigentlich sprengen. Auch die Figur des Guru kann nur im einzelnen Fall präzise verstanden werden. Kabir kann religionswissenschaftlich in seiner Rezeptionsgeschichte als eine Gründerfigur gesehen werden, aber er müsste eigentlich eher als Vermittler des Unfassbaren und Unübermittelbaren bezeichnet werden. Hingegen stellt Sundardas, ein Jahrhundert später, die schriftliche Tradition im weitesten Sinn als den eigentlichen Guru dar. Kabir konnte weltweit exportiert werden (wenn auch nicht à la lettre), während Dadu nur lokal bekannt ist und Sundardas nur unter Literaten.

Die beiden Beispiele von Kabir und Sundardas haben gezeigt, dass in der Sant-Bewegung, welche unter dieser Bezeichnung als immer dieselbe empfunden wird, für die Lehrer-Schüler-Genealogie verschiedene Regelungen und Sichtweisen verwirklicht wurden. Kabir lehnte eine Nachfolgeregelung überhaupt ab und wollte kein Guru sein; seine Nachfolger machten ihn jedoch zu einem Meister, der für eine Art Ökumenismus zwischen Hindus und Moslems eintritt, der als Retter vor dem Gesetz der Kaste (und damit heutzutage als Held der Subaltern Studies) betrachtet wird und den seine Nachfolger, die wie der populäre Hinduismus Regeln und Richtlinien angenommen haben, trotz und gegen die Überzeugungen Kabirs als Gründer sehen. Sukzession geschieht hier aufgrund eines Bruchs im Überlieferungszusammenhang mit dem, was der Gründer verkündete 
und verkörperte. Was die Gründergestalt trotzdem repräsentiert, ist eine Religiosität, die allen zugänglich ist. Dies steht in deutlichem Kontrast zu Kabirs Worten, die des Öfteren darauf hinweisen, dass sein Weg der schwerste, der gefährlichste und der am wenigsten begangene sei. Die innere Erfahrung des śabda kann kaum in Worten überliefert werden, die Schlagwörter seiner sozialen Aspekte und radikalen Offenheit können für jedes politische Programm verwendet werden.

Bei Sundardas beruht die Autorität auf seinem Gelehrtenwissen über die Wissenstraditionen und seiner Fähigkeit, damit den eigenen panth zu stützen und zu stärken. Er mag eigene Erfahrungen gemacht haben, aber er präsentiert sich als intellektueller Kompilator verschiedener Strömungen, der sich (dadurch) von den Sichtweisen des Meisters entfernt hat. Trotzdem bleibt dem Dadupanth, Dadus Aussprüchen und Lehren ebenso wie den späteren Traktaten und Hymnen des Sundardas die Bekanntheit und Breitenwirkung eines Kabir versagt. War 100 Jahre nach Kabir der Markt wieder oder noch gesättigt? Geht es um Charisma oder um Strategien der Sukzession? Lassen sich der Erfolg von Überlieferung überhaupt messen und vergleichen?

Mit diesen beiden Beispielen haben wir zwei verschiedene Vorstellungen vom Meister bzw. Guru kennen gelernt; Sukzession und Überlieferung funktionieren sehr verschieden als mündliche Mitteilung (bei Kabir) und als schriftliche Kommunikation (bei Sundardas). Die globalen Termini wie Hinduismus, Sant-Bewegung und Guru sind nur zu einem gewissen Grad nützlich; das Studium konkreter Beispiele von Sukzession verlangt nach komparativer Bearbeitung der einzelnen Aspekte. 


\section{Literatur}

Agrawal, Purushottam, Akath kahānī prem kī. Kabīr kī kavitā aur unkā samay, New Delhi:

Rajkamal Prakashan, 2010.

Agrawal, Purushottam, »In Search of Ramanand. The Guru of Kabir and Others «, in: Ishita

Banerjee Dube und Saurabh Dube (Hg.), Ancient to Modern. Religion, Power and

Community in India, New York: Oxford University Press, 2009, 135-170.

Agrawal, Purushottam, »Something Will Ring ... Translating Kabir and His Life«, in: Maya Burger und Nicola Pozza (Hg.), India in Translation through Hindi Literature. A Plurality of Voices,

Bern: Lang, 2010, 181-193.

Bahuguna, R.P., »Conflict and Assimilation in Medieval North Indian Bhakti. An Alternative

Approach «, http://jmi.ac.in/upload/departments/history/drs/Conflict\%20And\%20

Assimilation\%20In\%20Medieval\%20North\%20Indian\%20Bhakti.pdf (November 2015).

Brill's Encyclopedia of Hinduism, hg. von Knut A. Jacobsen, 6 Bde., Leiden: Brill, 2009-2015.

Bryant, Kenneth E., »Introduction. The Text«, in: Kenneth E. Bryant und John S. Hawley

(Hg.), Surdas. Sur's Ocean. Poems from the Early Tradition, Cambridge, Mass.: Harvard

University Press, 2015, xxiv-xli.

Bryant, Kenneth E. und John S. Hawley (Hg.), Surdas. Sur's Ocean. Poems from the Early

Tradition, Cambridge, Mass.: Harvard University Press, 2015.

Burger, Maya, Art. »Kabīr«, Brill’s Encyclopedia of Hinduism 4 (2014), 265-271.

Burger, Maya, Art. »Kabīrpanthīs«, Brill's Encyclopedia of Hinduism 3 (2011), 339-345.

Burger, Maya, "La Sarvāñga yoga pradīpaka de Sundardās. Une classification du yoga au 17e siècle", Asiatische Studien / Etudes asiatiques 68,3 (2014), 683-708.

Callewaert, Winand, The Millenium Kabīr Vānī. A Collection of Pad-s, New Delhi: Manohar, 2000.

Caturvedi, Parasuram, Uttarī bhārat kī sant paramparā, Allahabad: Bharati Bhandar, 1972.

Dharwadkar, Vinay, Kabir. The Weaver's Songs, New Delhi: Penguin Books, 2003.

Dvivedī, Kedaranath, Kabīr aur Kabīr Panth, Prayag, 1965 (Nachdruck Allahabad: Hindi Sahitya

Sammelan, 1995).

Hawley, John S., "Author and Authority in the Bhakti Poetry of North India«, Journal of Asian

Studies 47,2 (1988), 269-290.

Hawley, John S., Three Bhakti Voices. Mirabai, Surdas, and Kabir in Their Times and Ours, New

Delhi: Oxford India, 2005.

Hess, Linda, »Three Kabir Collections. A Comparative Study«, in: Karine Schomer und William H.

MacLeod (Hg.), The Sants. Studies in a Devotional Tradition of India, Berkeley: South Asia

Books, 1987, 111-141.

Hess, Linda und Sukhdev Singh, The Bijak of Kabir (1983), Delhi: Oxford University Press, 2002. Horstmann, Monika, »Hazārī Prasād Dvivedī’s Kabīr«, in: dies. (Hg.), Images of Kabīr, Delhi:

Manohar, 2002, 115-126.

Horstmann, Monika (Hg.), Images of Kabīr, Delhi: Manohar, 2002.

Gold, Daniel, The Lord as Guru. Hindi Sants in North Indian Tradition, New York: Oxford University Press, 1987.

Lorenzen, David N., »Dissent in Kabir and the Kabirpanth «, in: Vijaya Ramaswamy (Hg.), Devotion and Dissent in Indian History, New Delhi: Foundation Books, 2014, 169-187. Lorenzen, David N., Kabir Legends and Antanta-das's Kabir Parachai, Albany, N.Y.: State University of New York Press, 1991. 
Lorenzen, David N., »The Kabir-Panth and Social Protest«, in: Karine Schomer und William H. MacLeod (Hg.), The Sants. Studies in the Devotional Tradition of India, Berkeley: South Asia Books, 1987, 281-303.

Lorenzen, David N., »Traditions of Non-Caste Hinduism. The Kabir Panth«, Contributions to Indian Sociology 21 (1987), 264-283.

Lorenzen, David N., Who Invented Hinduism. Essays on Religion in History, New Delhi: Yoda Press, 2006.

Malinar, Angelika, Art. "Sampradāya«, Brill’s Encyclopedia of Hinduism 3 (2011), 339-345.

Misra, Ramasecandra, Sundaradāsa granthavalī, New Delhi: Kitab Ghar, 1992.

Pollock, Sheldon, „Comparison without Hegemony«, in: Hans Joas und Barbro Klein (Hg.), The Benefit of Broad Horizons. Intellectual and Institutional Preconditions for a Global Social Science, Leiden: Brill, 2010, 185-204.

Singh, Sukhdev, Kabīr Bījak, Allahabad: Nilabh Prakashan, 1972.

Steinmann, Ralph, Guru Śișiya Saṃbandha. Das Meister-Schüler-Verhältnis im traditionellen und modernen Hinduismus, Stuttgart: Steiner, 1986.

Suthren Hirst, Jacqueline und John Zavos, Religious Traditions in Modern South Asia, London: Routledge, 2011.

Thiel-Horstmann, Monika, Crossing the Ocean of Existence. Braj Bhāṣā Religious Poetry from Rajasthan, Wiesbaden: Harrassowitz, 1983.

Thukral, Uma, Kabīrpanth. Sāhitya, darśan, evam sādhanā, New Delhi: Hindi Book Center, 1998.

Vaudeville, Charlotte, Au cabaret de l'amour. Paroles de Kabîr, Paris: Gallimard, 1959.

Vaudeville, Charlotte, Kabīr, New Delhi: Oxford University Press, 1974 (Nachdruck als A Weaver Named Kabir, New Delhi: Oxford University Press, 1998).

Vaudeville, Charlotte, Kabīr Granthāvalī (doha), Pondichéry: Institut français d'Indologie, 1957.

Vaudeville, Charlotte, Kabīr-Vānī. Recension occidentale / Western Recension, Pondichéry: Institut français d'Indologie, 1982.

Venuti, Lawrence, The Translation Studies Reader, London: Routledge, 2012.

Venuti, Lawrence, "Translation, Community, Utopia«, in: ders., The Translation Studies Reader, London: Routledge, 2012, 468-509.

Weber, Max, Wirtschaft und Gesellschaft. Grundriss der verstehenden Soziologie (1922), Tübingen: Mohr Siebeck, 2002. 
